\title{
Outcomes of Endoscopic Submucosal Dissection for Early Gastric Cancer with Undifferentiated-Type Histology: A Clinical Simulation Using a Non- Selected Surgical Cohort
}

\author{
Dong Shin Kwak ${ }^{1}$, Yang Won Min ${ }^{1}$, Jun Haeng Lee ${ }^{1}$, Soo Hoon Kang ${ }^{1}$, Seung Hyeon Jang ${ }^{1}$, Hyuk Lee ${ }^{1}$, Byung-Hoon Min ${ }^{1}$, \\ Jae J. Kim ${ }^{1}$, Kyoung-Mee Kim ${ }^{2}$, Tae Sung Sohn ${ }^{3}$, and Sung Kim ${ }^{3}$ \\ Departments of ${ }^{1}$ Medicine, ${ }^{2}$ Pathology, and ${ }^{3}$ Surgery, Samsung Medical Center, Sungkyunkwan University School of Medicine, Seoul, Korea
}

Background/Aims: Outcomes of endoscopic submucosal dissection (ESD) for undifferentiated-type early gastric cancer (EGC) need to be further evaluated. We aimed to simulate the outcomes of ESD for undifferentiated-type EGC from a surgical database. Methods: Among 802 patients who underwent gastrectomy with endoscopic biopsy for poorly differentiated adenocarcinoma (PD-type) or signet ring cell carcinoma (SRC-type), ESD candidates meeting the expanded indication $(n=280)$ were selected by reviewing the endoscopic images. According to the surgical pathologic results, the outcomes of the ESD simulation were evaluated. Results: Among the candidates, 104 (37.1\%) were PD-type and 176 (62.9\%) were SRC-type. The curative resection (CR) rate was $42.1 \%$. Among the patients with $\mathrm{CR}$, three patients (2.5\%) showed lymph node metastasis (LNM). Three EGCs with CR and LNM were mucosal cancers $\geq 1.0 \mathrm{~cm}$ in size. The CR rate was higher in the SRC-type than in the PD-type (48.3\% vs $31.7 \%$, respectively, $p=0.007$ ). In the SRC-type, the $\mathrm{CR}$ rate was increased, with a smaller size criterion for the ESD indication, but was similar between the $1.0 \mathrm{~cm}$ and $0.6 \mathrm{~cm}$ criteria (63.3\% and $63.6 \%$, respectively), whereas the CR rate was below $50 \%$ in all of the different tumor size criteria (2.0 to $0.6 \mathrm{~cm}$ ) in the PD-type. Conclusions: In undifferentiated-type EGC, ESD should be considered in selected patients with tumor sizes $<1 \mathrm{~cm}$ and SRC histology. (Gut Liver 2018;12:263-270)

Key Words: Early gastric cancer; Endoscopic mucosal resection; Undifferentiated

\section{INTRODUCTION}

Endoscopic submucosal dissection (ESD) has been established as a standard treatment of early gastric cancers (EGCs) meeting the absolute indication. In addition, expanded indications were proposed by Gotoda et al. ${ }^{1}$ based on detailed pathologic analysis of surgical database about the risk factors of lymph node (LN) metastasis. Recent guidelines include expanded indications for possible candidate of endoscopic treatment of EGC, although Japanese guidelines consider them experimental. ${ }^{2,3}$ Expanded indications can be divided into two subgroups, differentiatedtype and undifferentiated-type EGC. ${ }^{4}$ Although clinical outcomes of endoscopic treatment for differentiated-type EGC meeting expanded indications were excellent, ${ }^{5-10}$ it remains unclear for undifferentiated-type EGC.

Although there have been a number of studies about ESD for undifferentiated-type EGC, ${ }^{11-16}$ they have an important limitation of selection bias. Most of them are small retrospective studies with unclearly defined inclusion criteria. Patients were usually enrolled when the final histology after ESD was undifferentiated-type histology, either poorly differentiated adenocarcinoma (PD-type) or signet ring cell carcinoma (SRCtype). The initial endoscopic biopsy-proven histology prior to ESD, however, was heterogeneous. Many studies thus included patients who showed differentiated-type EGC on the forceps biopsy and undifferentiated-type EGC on the final ESD pathology. ${ }^{12,16}$ Indeed, most cases with undifferentiated-type histology were not prospectively selected by expanded indications but were retrospectively collected by the final ESD pathology.

In our institution, patients with endoscopic biopsy-proven undifferentiated-type gastric cancer underwent surgery. In order

\footnotetext{
Correspondence to: Jun Haeng Lee

Department of Medicine, Samsung Medical Center, Sungkyunkwan University School of Medicine, 81 Irwon-ro, Gangnam-gu, Seoul 06351, Korea Tel: +82-2-3410-3409, Fax: +82-2-3410-6983, E-mail: stomachlee@gmail.com Received on June 7, 2017. Revised on July 19, 2017. Accepted on August 18, 2017. Published online December 26,2017 pISSN 1976-2283 eISSN 2005-1212 https://doi.org/10.5009/gnl17247 Dong Shin Kwak and Yang Won Min contributed equally to this work as first authors.

@ This is an Open Access article distributed under the terms of the Creative Commons Attribution Non-Commercial License (http://creativecommons.org/licenses/by-nc/4.0) which permits unrestricted non-commercial use, distribution, and reproduction in any medium, provided the original work is properly cited.
} 
to avoid selection bias, we selected ESD candidates (feasible for ESD) meeting expanded indications from the surgical cohort of endoscopically suspected undifferentiated-type EGC on the forceps biopsy. Using these ESD candidates, we simulated ESD outcomes with reference to surgical pathologic results. In addition, we assessed the outcomes of simulated ESD with various size criteria of expanded indication and with a different curative resection (CR) size definition to suggest the optimal size criteria for ESD in undifferentiated-type EGC.

\section{MATERIALS AND METHODS}

\section{Subjects}

Between January 2014 and December 2014, subtotal gastrectomy or total gastrectomy for gastric cancer was performed in a total of 1,336 consecutive patients at Samsung Medical Center, Seoul, Korea. Among them, we identified 802 patients who had undifferentiated-type (PD-type or SRC-type) gastric cancer on the result of endoscopic forceps biopsy. From them, we included 537 patients whose endoscopic diagnosis was EGC. Endoscopic images of the 537 patients were reviewed by an ESD expert (J.H.L.) to select ESD candidates meeting expanded indications (tumor with a diameter of $20 \mathrm{~mm}$ or smaller, confined to mucosa, and without ulceration). Findings of abdomen computed tomography were used as a reference to exclude cases with distant metastasis or regional LN metastasis. Finally, 280 EGCs seemed to be feasible for ESD and underwent ESD simulation (Fig. 1). This study protocol was conducted in accordance with the Declaration of Helsinki and approved by the Institutional Review Board (IRB) at Samsung Medical Center, Seoul, Korea (IRB number: 2015-06-056-001). The IRB waived the requirement for informed consent, because we used de-identified data.

\section{Data collection}

The following information were collected from each patient: age, sex, endoscopic tumor size, tumor location, endoscopic tumor gross type, pathologic tumor size, final tumor pathology, depth of tumor invasion, and presence of LN metastasis on pathology. Endoscopic tumor size was defined as the maximal tumor size at endoscopy and pathologic tumor size was measured upon the microscopic examination from the resected specimen. Tumor locations were categorized by longitudinal axis of the stomach. The axis was divided into three sections (the upper third containing the fundus, cardia, and upper body, the middle third containing the mid-body, lower body, and angle, and the lower third containing the antrum and pylorus). ${ }^{17}$ Tumor gross types were classified by their predominant type. The protruded type and superficial elevated type were classified as elevated type. The superficial flat type was classified as flat type, and the superficial depressed type and excavated type were classified as depressed type. ${ }^{18}$

The fixed ESD specimen was sectioned serially at 2-mm intervals, parallel to a line that included the closest resection margin of the specimen so that both lateral and vertical margins could be assessed. The depth of tumor invasion was then evaluated along with lymphovascular invasion and differentiation. However, the surgical specimen was sectioned serially at 4-mm intervals.

\section{Outcomes of simulated ESD}

According to the final pathologic result of surgical specimen, outcomes of simulated ESD were evaluated. CR rate was assessed on the assumption that ESD was done by en bloc resection without technical failure. CR of undifferentiated-type EGC was defined when tumor was smaller than $2 \mathrm{~cm}$, confined to mucosa lesion, without ulceration, and without lymphovascular invasion in the surgical specimens. ${ }^{19}$

In addition, $\mathrm{CR}$ rate was assessed when different size criteria $(1.5,1.0$, and $0.6 \mathrm{~cm})$ of expanded ESD indication for undifferentiated-type EGC were applied. Modified CR of undifferentiated-type EGC defined when tumor was smaller than $3 \mathrm{~cm}$, confined to mucosa lesion, and without ulceration in the surgical specimens was also assessed.

\section{Statistical analysis}

Statistical results are presented as the mean \pm standard deviation or number of patients (\%). Continuous variables were com-

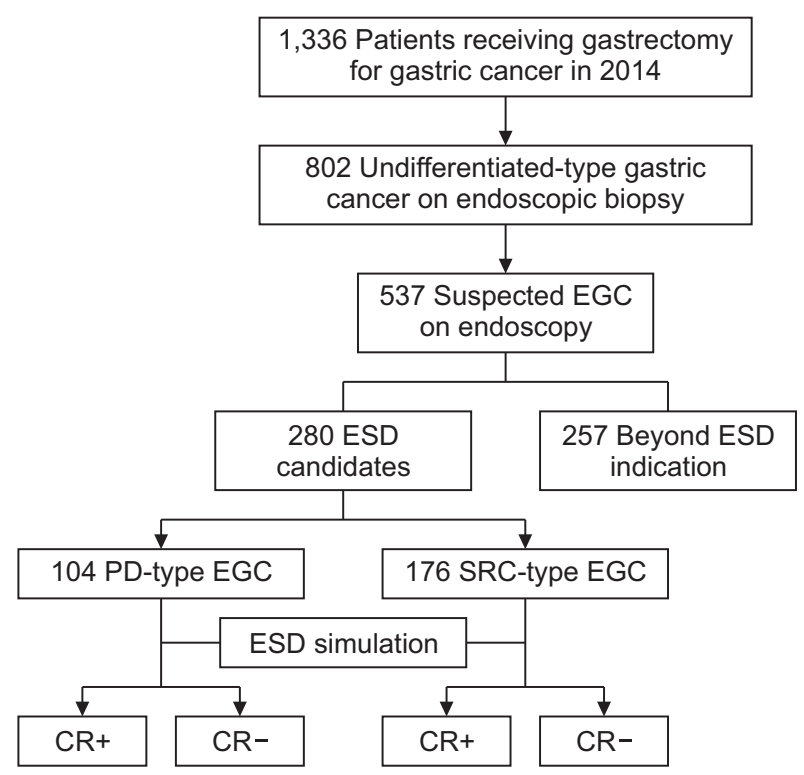

Fig. 1. Subject flow. Among the 1,336 gastric cancer patients undergoing gastrectomy in 2014, 802 undifferentiated-type gastric cancer patients were identified based on endoscopic biopsy. Among them, 537 patients whose endoscopic diagnosis was early gastric cancer (EGC) were included. By reviewing endoscopic images of the 537 patients, we selected endoscopic submucosal dissection (ESD) candidates meeting the expanded indications. The curative resection (CR) rate was assessed on the assumption that the ESD was performed by en bloc resection without technical failure.

PD, poorly differentiated; SRC, signet ring cell. 
pared parametrically using Student t-test. Categorical variables were compared using the chi-square test or Fisher exact test as appropriate. Two-sided p-values $<0.05$ were taken as statistically significant. Statistical analyses were conducted using the SPSS Statistics version 21.0 software (IBM Corp., Armonk, NY, USA).

\section{RESULTS}

\section{Characteristics of endoscopically suspected undifferenti- ated-type EGCs}

Table 1 shows the characteristics of endoscopically suspected EGC with undifferentiated-type histology on the forceps biopsy. Among the 537 EGCs, 206 (38.3\%) were PD-type and 331 (61.7\%) were SRC-type. The mean endoscopic tumor size was $2.17 \pm 1.41 \mathrm{~cm}$. Ulceration was observed in $95(17.7 \%)$ tumors at endoscopy. Tumor location was upper in 60 (11.1\%), middle in 316 (58.9\%), and lower stomach in 161 (30.0\%). The tumor gross type was flat/depressed in 486 (90.5\%) and elevated in 51

Table 1. Baseline Characteristics of Endoscopically Suspected Undifferentiated-Type Early Gastric Cancer

\begin{tabular}{lcccc}
\hline & $\begin{array}{c}\text { Total } \\
(\mathrm{n}=537)\end{array}$ & $\begin{array}{c}\text { PD-type } \\
(\mathrm{n}=206)\end{array}$ & $\begin{array}{c}\text { SRC-type } \\
(\mathrm{n}=331)\end{array}$ & p-value* \\
\hline Age, yr & $52.9 \pm 11.2$ & $54.0 \pm 11.8$ & $52.2 \pm 10.8$ & 0.079 \\
Male sex & $258(48.0)$ & $105(51.0)$ & $153(46.2)$ & 0.284 \\
Endoscopic tumor size, cm & $2.17 \pm 1.41$ & $2.21 \pm 1.41$ & $2.14 \pm 1.41$ & 0.613 \\
Ulceration at endoscopy & $95(17.7)$ & $39(18.9)$ & $56(16.9)$ & 0.563 \\
Pathologic tumor size, cm & $3.03 \pm 2.05$ & $3.15 \pm 1.99$ & $2.95 \pm 2.09$ & 0.275 \\
Size discrepancy, cm & $-0.85 \pm 1.69-0.94 \pm 1.60-0.80 \pm 1.75$ & 0.366 \\
Final pathology & & & & $<0.001$ \\
$\quad$ MD & $38(7.0)$ & $28(13.6)$ & $10(3.0)$ & \\
$\quad$ PD & $269(50.0)$ & $153(74.3)$ & $116(35.0)$ & \\
$\quad$ SRC & $230(43.0)$ & $25(12.1)$ & $205(62.0)$ & \\
Location & & & & 0.956 \\
$\quad$ Upper & $60(11.1)$ & $24(11.7)$ & $36(10.9)$ & \\
$\quad$ Middle & $316(58.9)$ & $120(58.3)$ & $196(59.2)$ & \\
$\quad$ Lower & $161(30.0)$ & $62(30.0)$ & $99(29.9)$ & \\
Gross type & & & & 0.022 \\
$\quad$ Flat/depressed & $486(90.5)$ & $194(94.2)$ & $292(88.2)$ & \\
Elevated & $51(9.5)$ & $12(5.8)$ & $39(11.8)$ & \\
Depth of invasion & & & & 0.539 \\
$\quad$ Mucosa & $332(61.8)$ & $124(60.2)$ & $208(62.8)$ & \\
$\quad$ Submucosa and beyond & $205(38.2)$ & $82(39.8)$ & $123(37.2)$ & \\
Lymphovascular invasion & $83(15.5)$ & $39(18.9)$ & $44(13.3)$ & 0.079 \\
LN metastasis & $73(13.6)$ & $28(13.6)$ & $45(13.9)$ & 0.988 \\
\hline Data are presnted as & & & \\
\hline
\end{tabular}

Data are presented as mean \pm SD or the number $(\%)$.

$\mathrm{PD}$, poorly differentiated; SRC, signet ring cell; MD, moderately differentiated; LN, lymph node.

*Comparison between the PD-type and the SRC-type.
(9.5\%). The mean pathologic tumor size was $3.03 \pm 2.05 \mathrm{~cm}$ and size discrepancy between endoscopic estimation and surgical specimen was $-0.85 \pm 1.69 \mathrm{~cm}$. According to the final pathologic results, 38 EGCs (7.0\%) were differentiated-type EGCs (all moderately differentiated), 269 (50.0\%) were PD-type EGCs, and 230 (43.0\%) were SRC-type EGCs; 332 (61.8\%) were confined to the mucosal layer, 168 (31.3\%) were invading into the submucosal layer, and 37 (6.9\%) were beyond the submucosal layer; 83 (15.5\%) had lymphovascular invasion; LN metastasis was found in 73 EGCs (13.6\%). These characteristics of undifferentiatedtype EGC did not differ by endoscopic forceps biopsy-proven histology (PD-type vs SRC-type) except for the tumor gross type and final histologic type. PD-type EGD had more frequent flat/ depressed type (94.2\% vs $88.2 \%, p=0.022)$ and showed more frequent histologic discrepancy $(\mathrm{p}<0.001)$ than SRC-type EGC.

\section{Characteristics of ESD candidate EGCs}

Table 2 shows the characteristics of ESD candidate EGCs $(n=280)$ meeting expanded indications among the endoscopically suspected undifferentiated-type EGCs. The mean age was $52.5 \pm 10.9$ years and 131 patients $(46.0 \%)$ were male. The mean

Table 2. Comparison of the Characteristics between ESD Candidates and Beyond ESD Indication

\begin{tabular}{|c|c|c|c|}
\hline & $\begin{array}{c}\text { Expanded } \\
\text { indication } \\
(\mathrm{n}=280)\end{array}$ & $\begin{array}{l}\text { Beyond ESD } \\
\text { indication } \\
(\mathrm{n}=257)\end{array}$ & $\mathrm{p}$-value \\
\hline Age, yr & $52.5 \pm 10.9$ & $53.35 \pm 11.5$ & 0.385 \\
\hline Male sex & $131(46.8)$ & $127(49.4)$ & 0.542 \\
\hline Endoscopic tumor size, $\mathrm{cm}$ & $1.21 \pm 0.46$ & $3.21 \pm 1.36$ & $<0.001$ \\
\hline Ulceration at endoscopy & 0 & $95(37.0)$ & $<0.001$ \\
\hline Pathologic tumor size, $\mathrm{cm}$ & $2.13 \pm 1.29$ & $4.01 \pm 2.27$ & $<0.001$ \\
\hline Size discrepancy, $\mathrm{cm}$ & $-0.92 \pm 1.24$ & $-0.79 \pm 2.07$ & 0.395 \\
\hline Pathology & & & 0.544 \\
\hline PD & $104(37.1)$ & $102(39.7)$ & \\
\hline SRC & $176(62.9)$ & $155(60.3)$ & \\
\hline Location & & & $<0.001$ \\
\hline Upper & $16(5.8)$ & $44(17.1)$ & \\
\hline Middle & $166(59.2)$ & $150(58.3)$ & \\
\hline Lower & $98(35.0)$ & $63(24.5)$ & \\
\hline Gross type & & & 0.678 \\
\hline Flat/depressed & $252(90.0)$ & 234 (91.0) & \\
\hline Elevated & $28(10.0)$ & $23(9.0)$ & \\
\hline Depth of invasion & & & $<0.001$ \\
\hline Mucosa & $208(74.2)$ & $124(48.2)$ & \\
\hline Submucosa and beyond & $72(25.8)$ & $133(51.8)$ & \\
\hline Lymphovascular invasion & $22(7.8)$ & $61(23.7)$ & $<0.001$ \\
\hline LN metastasis & $22(7.8)$ & 51 (19.8) & $<0.001$ \\
\hline
\end{tabular}

Data are presented as mean \pm SD or the number $(\%)$.

ESD, endoscopic submucosal dissection; PD, poorly differentiated; SRC, signet ring cell; LN, lymph node. 
endoscopic tumor size was $1.21 \pm 0.46 \mathrm{~cm}$. On the forceps biopsy, 104 (37.1\%) were PD-type and 176 (62.9\%) were SRC-type. Tumor location was upper in 16 (5.8\%), middle in 166 (59.2\%), and lower stomach in 98 (35.0\%). The endoscopic tumor gross type was flat/depressed in 252 (90.0\%) and elevated in 28 (10.0\%). The mean pathologic tumor size was $2.13 \pm 1.29 \mathrm{~cm}$. Size discrepancy between endoscopic estimation and surgical specimen was $-0.92 \pm 1.24 \mathrm{~cm}$. According to the final pathologic results, 208 EGCs (74.2\%) were confined to the mucosal layer and $72(25.8 \%)$ were invading into the submucosal layer; LN metastasis was found in 22 (7.8\%).

There were no differences between ESD candidate EGCs $(n=280)$ and EGCs beyond ESD indication $(n=257)$ in terms of age, sex, size discrepancy, endoscopic gross type, and forceps biopsy histology. However, ulceration was observed at endoscopy in 95 (37.0\%) of beyond ESD indication group. In addition, ESD candidate group had smaller endoscopic and pathologic tumor sizes $(1.21 \pm 0.46 \mathrm{~cm}$ and $2.13 \pm 1.29 \mathrm{~cm}$ vs $3.21 \pm 1.36 \mathrm{~cm}$ and $4.01 \pm 2.27 \mathrm{~cm}$, all $\mathrm{p}<0.001)$, less frequent upper stomach and more frequent lower stomach location (5.8\% and 35.0\% vs $17.1 \%$ and $24.5 \%, p<0.001$ ), less frequent submucosal tumor invasion ( $25.8 \%$ vs $51.8 \%, \mathrm{p}<0.001$ ), less frequent lymphovascular invasion (7.8\% vs 23.7\%, $\mathrm{p}<0.001)$, and less frequent LN metastasis $(7.8 \%$ vs $19.8 \%, \mathrm{p}<0.001)$ than beyond ESD indication group.

\section{Outcomes of simulated ESD}

CR rate was 42.1\% (118) in the ESD candidate EGCs $(n=280)$. CR rate was lower in PD-type EGC than in SRC-type EGC (31.7\% vs $48.3 \%, p=0.007)$. Causes of non-CR $(n=162)$ were as follows: tumor size $>2 \mathrm{~cm}$ in diameter in 121 (74.7\%), submucosa or beyond tumor invasion in 72 (44.5\%), and lymphovascular invasion in 22 (13.6\%). According to the endoscopic forceps biopsy-proven histology, causes of non-CR were as follows: tu-

Table 3. Comparison of the Characteristics between the CR and NonCR Groups among the Endoscopic Submucosal Dissection Candidates Meeting the Expanded Criteria

\begin{tabular}{lccr}
\hline & $\mathrm{CR}(\mathrm{n}=118)$ & Non-CR $(\mathrm{n}=162)$ & $\mathrm{p}$-value \\
\hline Age, yr & $51.9 \pm 10.3$ & $53.0 \pm 11.3$ & 0.847 \\
Male sex & $56(47.4)$ & $75(46.2)$ & 0.421 \\
Endoscopic tumor size, cm & $1.08 \pm 0.43$ & $1.30 \pm 0.46$ & $<0.001$ \\
Pathologic tumor size, cm & $1.22 \pm 0.45$ & $2.78 \pm 1.30$ & $<0.001$ \\
Size discrepancy, cm & $-0.14 \pm 0.43$ & $-1.48 \pm 1.33$ & $<0.001$ \\
Pathology & & & 0.007 \\
$\quad$ PD & $33(28.0)$ & $71(45.6)$ & \\
$\quad 35 C$ & $85(72.0)$ & $91(54.4)$ & \\
Location & & & 0.080 \\
$\quad$ Upper & $3(2.5)$ & $13(8.0)$ & \\
$\quad$ Middle & $68(57.6)$ & $98(60.5)$ & \\
$\quad$ Lower & $47(39.9)$ & $51(31.5)$ & \\
Gross type & & & 0.747 \\
$\quad$ Flat/depressed & $107(90.6)$ & $145(89.5)$ & \\
$\quad$ Elevated & $11(9.4)$ & $17(10.5)$ & \\
Depth of invasion & & & $<0.001$ \\
$\quad$ Mucosa & $118(100)$ & $90(55.5)$ & \\
$\quad$ Submucosa and beyond & 0 & $72(44.5)$ & \\
Lymphovascular invasion & 0 & $22(13.6)$ & $<0.001$ \\
LN metastasis & $3(2.5)$ & $19(11.7)$ & 0.004 \\
\hline
\end{tabular}

Data are presented as mean \pm SD or the number $(\%)$.

$\mathrm{CR}$, curative resection; $\mathrm{PD}$, poorly differentiated; SRC, signet ring cell; LN, lymph node.
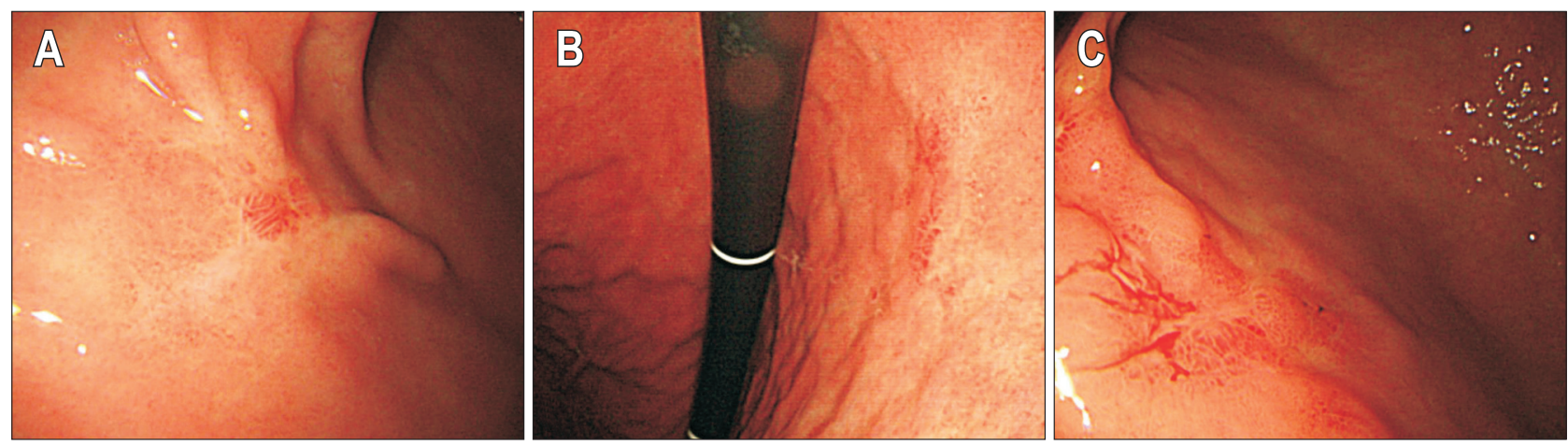

Fig. 2. Esophagogastroduodenoscopy appearances of three undifferentiated-type early gastric cancers meeting the curative resection criteria and exhibiting lymph node (LN) metastasis. All were mucosal cancers with mixed histology of poorly differentiated adenocarcinoma (PD-type) with a signet ring cell carcinoma (SRC-type) component. (A) A 57-year-old female with a $1.3 \mathrm{~cm}$, ill-defined, pale, depressed lesion with abnormal converging folds, such as cutting and tapering on the anterior side of the angle. Endoscopic biopsy revealed the SRC-type, and the final result was a $1.5 \mathrm{~cm}$ mucosal cancer with metastasis to two out of 67 regional LNs. (B) A 64-year-old female with a $1.7 \mathrm{~cm}$, pale, depressed lesion with an erythematous tumor island on the posterior wall of the lower body. Endoscopic biopsy indicated the PD-type, and the final result was a $1.1 \mathrm{~cm}$ mucosal cancer with metastasis to one out of 42 regional LNs. (C) A 46-year-old female with a $1.0 \mathrm{~cm}$, pale, geographic, depressed lesion with an erythematous tumor island on the antero-greater curvature side of the proximal antrum. Endoscopic biopsy revealed the PD-type, and the final result was a $1.4 \mathrm{~cm}$ mucosal cancer with metastasis to two out of 37 regional LNs. 


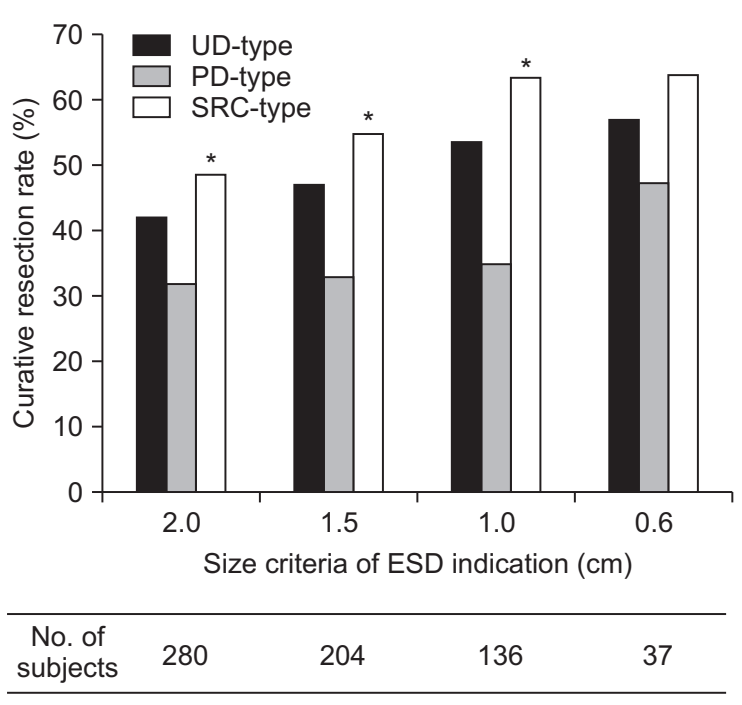

Fig. 3. Number of endoscopic submucosal dissection (ESD) candidates and their curative resection rate according to the different size criteria of the ESD indication.

UD, undifferentiated; PD, poorly differentiated; SRC, signet ring cell. ${ }^{*} \mathrm{p}<0.05$ on comparison between PD-type and SRC-type.

mor size $>2 \mathrm{~cm}$ in diameter in 51 (71.8\%) of PD-type and in 70 (76.9\%) of SRC-type, submucosal or beyond tumor invasion in 35 (49.3\%) of PD-type and in 37 (40.7\%) of SRC-type, and lymphovascular tumor invasion in 12 (16.9\%) of PD-type and in 10 (11.0\%) of SRC-type.

In Table 3, characteristics were compared between CR and non-CR groups. There were no differences in terms of age, sex, tumor location, and endoscopic tumor gross type between the two groups. However, CR group had smaller endoscopic and pathologic tumor sizes and size discrepancy $(1.08 \pm 0.43 \mathrm{~cm}$, $1.22 \pm 0.45 \mathrm{~cm}$, and $-0.14 \pm 0.43 \mathrm{~cm}$ vs $1.30 \pm 0.46 \mathrm{~cm}, 2.78 \pm 1.30$ $\mathrm{cm}$, and $-1.48 \pm 1.33 \mathrm{~cm}$, respectively; all $\mathrm{p}<0.001$ ), lower frequent PD-type EGC (28.0\% vs $45.6 \%, \mathrm{p}=0.007)$, and less frequent LN metastasis (2.5\% vs $11.7 \%, \mathrm{p}=0.004)$ than non-CR group. Among the three EGCs with CR and LN metastasis, two were PD-type and one was SRC-type and all were mucosal cancers larger than $1.0 \mathrm{~cm}$ in size (Fig. 2).

When CR size criterion was modified from $2 \mathrm{~cm}$ to $3 \mathrm{~cm}, \mathrm{CR}$ rate was 60.7\% (170/280). However, LN metastasis was found in five (2.9\%) patients with CR. According to the endoscopic forceps biopsy-proven histology, CR rate was higher in SRC-type EGC than in PD-type EGC (68.7\% vs $47.1 \%, \mathrm{p}<0.001)$ while rate of $\mathrm{LN}$ metastasis did not differ significantly (1.7\% vs $6.1 \%$, $\mathrm{p}=0.145$ ) between SRC-type and PD-type EGC groups.

\section{Outcomes of simulated ESD for smaller EGCs}

In Fig. 3, number of ESD candidates and their CR rate according to different size criteria $(2.0,1.5,1.0$, and $0.6 \mathrm{~cm})$ of ESD indication for undifferentiated-type EGC are shown. When a size criterion of $1.5 \mathrm{~cm}$ was applied, number of ESD candidates was decreased by $27.1 \%$ (from 280 to 204) with CR rate of $47.1 \%$
(32.4\% in PD-type and 54.9\% in SRC-type, $\mathrm{p}=0.002$ ). Among the patients with $\mathrm{CR}$, LN metastasis was found in two patients (2.1\%, 2/96; one PD-type and one SRC-type) (Fig. 2). When 1.0 $\mathrm{cm}$, number of ESD candidates was decreased by $51.4 \%$ (to 136) with CR rate of 53.6\% (34.8\% in PD-type and 63.3\% in SRCtype, $p=0.002$ ). Among the patients with $\mathrm{CR}, \mathrm{LN}$ metastasis was found in one patients (1.4\%, 1/73; PD-type) (Fig. 2). When 0.6 $\mathrm{cm}$, number of ESD candidate was decreased by $86.8 \%$ (to 37 ) with CR rate of 56.8\% (46.7\% in PD-type and 63.6\% in SRCtype, $p=0.306$ ). Among the patients with $\mathrm{CR}, \mathrm{LN}$ metastasis was not found. In PD-type EGC, CR rate was below 50\% in all of different tumor size criteria $(2.0,1.5,1.0$, and $0.6 \mathrm{~cm})$. In SRCtype EGC, CR rate was increased with smaller size criterion of ESD indication and was similar between $1.0 \mathrm{~cm}$ and $0.6 \mathrm{~cm}$ criteria.

\section{DISCUSSION}

ESD has not been considered as a treatment option for undifferentiated-type EGC. As several studies showed LN metastasis is negligible in small undifferentiated-type mucosal lesion, ${ }^{20-23}$ undifferentiated-type EGC was included in the expanded ESD indication in recent guidelines as an investigational treatment. Although there have been several studies suggesting ESD as an alternative treatment option in undifferentiated-type EGC, ${ }^{13,14,24}$ large scaled long-term data of ESD is still insufficient. Furthermore, previous studies have a limitation of having selection bias as we discussed in the premise. To minimize the selection bias, we did ESD simulation using a non-selected surgical cohort and analyzed the outcomes according to the histopathological results on the assumption that ESD was done by en bloc resection without technical failure. CR rate of ESD simulation was calculated as $42.1 \%$. However, CR rate was higher in SRC-type EGC than in PD-type EGC (48.3\% vs 31.7\%, p=0.007). In SRCtype EGC, CR rate was increased with smaller size criterion of ESD indication and was similar between $1.0 \mathrm{~cm}$ and $0.6 \mathrm{~cm}$ criteria while $\mathrm{CR}$ rate was below 50\% in all of different tumor size criteria $(2.0,1.5,1.0$, and $0.6 \mathrm{~cm})$ in PD-type EGC. In addition, no LN metastasis was found when CR was achieved in tumor smaller than $1 \mathrm{~cm}$ in size. Thus we could suggest that ESD for undifferentiated-type EGC should be considered in more selected patients, such as tumor size smaller than $1 \mathrm{~cm}$ with histology of SRC to achieve better outcomes.

In the previous studies, long-term outcomes of ESD for undifferentiated-type EGC are good when CR is achieved. ${ }^{14,15,25} \mathrm{CR}$ rates of ESD simulation in the present study are consistent with that of the previous real ESD studies. ${ }^{15,25}$ In the recent retrospective study, ${ }^{25} \mathrm{CR}$ was also achieved more frequently in SRC-type EGC (61.4\%) than in PD-type EGC (45.1\%) after ESD for undifferentiated-type EGC. In our results, common causes of non$\mathrm{CR}$ are tumor size $>2 \mathrm{~cm}$ in diameter (74.7\%) and submucosa or beyond tumor invasion (44.4\%). To improve CR rate, accurate 
determination of depth and extent of tumor is thus necessary. Unfortunately, however, this can be difficult in undifferentiatedtype EGC. The accuracy of depth diagnosis by endoscopic ultrasound (EUS) in undifferentiated-type EGC has been known to be worse than in differentiated-type EGC. ${ }^{26}$ Undifferentiatedtype EGC can extend along the proliferative zone in the middle layer of the mucosa, leaving normal ducts covering the superficial epithelium. ${ }^{27}$ In this case, even magnifying endoscopy with narrow band imaging was less useful. ${ }^{28}$ In our results, larger tumor showed a tendency to be more underestimated in size on endoscopy while tumor $<1 \mathrm{~cm}$ in size was not underestimated (Supplementary Table 1). In addition, among ESD candidates prediction of tumor depth was accurate only in $67.8 \%$ when tumor is $>1 \mathrm{~cm}$ and $\leq 2 \mathrm{~cm}$ in size while $81.0 \%$ when $\leq 1 \mathrm{~cm}$. Taken together, application of ESD for small lesion less than $1 \mathrm{~cm}$ and pretreatment circumferential mapping biopsy could be optimal strategy for improving $\mathrm{CR}$ rate. Furthermore, wide marking beyond estimated lesion during ESD would be necessary to secure enough resection margins.

In the previous report, PD-type EGC has pathological features such as submucosal invasion, ulcer, and lymphatic invasion that are less favorable to endoscopic treatment than that of SRC-type EGC. ${ }^{19}$ We thus should take into account separate approach to these two types of EGC, not as a united type of undifferentiated-type histology. Indeed, CR rate was constantly below 50\% in various size criteria of ESD indication while CR rate was increased beyond $60 \%$ with smaller size criterion of ESD indication (1.0 and $0.6 \mathrm{~cm}$ ).

The most important factor concerning ESD with curative intent is the prediction of LN metastasis. However, the reported rate of LN metastasis in undifferentiated-type EGC ranges from $5.7 \%$ to $20 \%$ which is higher than that of differentiated-type EGC. ${ }^{29-33}$ Although Gotoda et al. ${ }^{1}$ reported that LN metastasis was not found in the undifferentiated-type EGC smaller than 2 $\mathrm{cm}$, confined to mucosa without ulceration, other studies proposed that expanded ESD indication for undifferentiated-type EGC should be narrowed to the smaller lesion. ${ }^{19,21,34,35}$ These are consistent with our data which show no EGC smaller than 1.0 cm with CR had LN metastases (Supplementary Table 2). Thus, pathologic expanded indications with $2.0 \mathrm{~cm}$ size criterion may not be a good endpoint to define the optimal selection criteria for ESD. Indeed, the rate of LN metastasis was 2.5\% in CR group. However, the risk of LN metastasis is 2.9\% and $1.6 \%$ even in the low risk group by the prediction model for SRC-type and PD-type, respectively. ${ }^{36,37}$ Therefore, expanded indication could be used to determine the feasible selection criteria for ESD in undifferentiated-type EGC. However, although the risk for LNM is very low, it should not be considered negligible in endoscopic resection.

Three EGCs (two PD-type and one SRC-type on endoscopic biopsy) with CR and LN metastasis had mixed histology with SRC component on the final pathologic results and were devel- oped in women in their 40s to 60s. Previous case reports with LN metastasis from undifferentiated-type mucosal gastric cancer satisfying the expanded criteria showed similar characteristics of middle age women with mixed histology. ${ }^{38,39}$ From these observations we could suggest that middle age woman with mixed histology needs more intense surveilance of recurrence although CR has been achieved.

The current study has some limitations. Firstly, ESD simulation was done on the assumption that ESD was done by en bloc resection without technical failure. Thus CR rate could be lower in real practice considering nonlifting, piecemeal resection, and complications during procedure. However, recent two studies reported the en bloc resection rate of 99\% in ESD for undifferentiated-type EGC. ${ }^{14,15}$ In addition, as wide marking of $10 \mathrm{~mm}$ beyond estimated lesion is usually applied in ESD for undifferentiated-type EGC difference in CR rate between ESD simulation and real ESD may not be significant. Second, preoperative evaluation using EUS was not done and could not be taken into account in our simulation. Although the role of EUS in predicting depth and extent of tumor is limited in undifferentiated-type EGC, preoperative EUS might enhance CR rate. Third, pathologic result of surgical specimen could underestimate depth of invasion and presence of lymphovascular invasion. Lastly, ESD candidates meeting expanded indications were selected from an ESD expert by reviewing endoscopic images. This may limit the generalizability of our results. Nevertheless, our study has strengths of minimized selection bias and large sample size. Because patients with endoscopic biopsy-proven undifferentiatedtype gastric cancer underwent surgery in our institution, we could avoid selection bias from the non-selected surgical cohort. Using ESD simulation methods, we could analyze relatively large samples of undifferentiated-type EGC. In conclusion, our ESD simulation with a non-selected surgical cohort results suggest that ESD should be considered in more selected patients, such as tumor size smaller than $1 \mathrm{~cm}$ with histology of SRC to achieve better outcomes in undifferentiated-type EGC.

\section{CONFLICTS OF INTEREST}

No potential conflict of interest relevant to this article was reported.

\section{ACKNOWLEDGEMENTS}

Author contributions: D.S.K. and Y.W.M. analyzed the data and wrote the manuscript; S.H.K. and S.H.J. collected data; H.L., B.H.M., J.J.K., K.M.K., T.S.S., and S.K. were involved in the study design and undertook critical revision of the manuscript; J.H.L. designed the study and revised the manuscript. 


\section{REFERENCES}

1. Gotoda T, Yanagisawa A, Sasako M, et al. Incidence of lymph node metastasis from early gastric cancer: estimation with a large number of cases at two large centers. Gastric Cancer 2000;3:219225.

2. Japanese Gastric Cancer Association. Japanese gastric cancer treatment guidelines 2010 (ver. 3). Gastric Cancer 2011;14:113123.

3. Lee JH, Kim JG, Jung HK, et al. Clinical practice guidelines for gastric cancer in Korea: an evidence-based approach. J Gastric Cancer 2014;14:87-104.

4. Park CH, Shin S, Park JC, et al. Long-term outcome of early gastric cancer after endoscopic submucosal dissection: expanded indication is comparable to absolute indication. Dig Liver Dis 2013;45:651-656.

5. Goto 0, Fujishiro M, Kodashima S, Ono S, Omata M. Outcomes of endoscopic submucosal dissection for early gastric cancer with special reference to validation for curability criteria. Endoscopy 2009;41:118-122.

6. Gotoda T, Iwasaki M, Kusano C, Seewald S, Oda I. Endoscopic resection of early gastric cancer treated by guideline and expanded National Cancer Centre criteria. Br J Surg 2010;97:868-871.

7. Lee H, Yun WK, Min BH, et al. A feasibility study on the expanded indication for endoscopic submucosal dissection of early gastric cancer. Surg Endosc 2011;25:1985-1993.

8. Ahn JY, Jung HY, Choi KD, et al. Endoscopic and oncologic outcomes after endoscopic resection for early gastric cancer: 1370 cases of absolute and extended indications. Gastrointest Endosc 2011;74:485-493.

9. Choi MK, Kim GH, Park DY, et al. Long-term outcomes of endoscopic submucosal dissection for early gastric cancer: a singlecenter experience. Surg Endosc 2013;27:4250-4258.

10. Kim YI, Kim YW, Choi IJ, et al. Long-term survival after endoscopic resection versus surgery in early gastric cancers. Endoscopy 2015;47:293-301.

11. Kamada K, Tomatsuri N, Yoshida N. Endoscopic submucosal dissection for undifferentiated early gastric cancer as the expanded indication lesion. Digestion 2012;85:111-115.

12. Kang HY, Kim SG, Kim JS, Jung HC, Song IS. Clinical outcomes of endoscopic submucosal dissection for undifferentiated early gastric cancer. Surg Endosc 2010;24:509-516.

13. Yamamoto Y, Fujisaki J, Hirasawa T, et al. Therapeutic outcomes of endoscopic submucosal dissection of undifferentiated-type intramucosal gastric cancer without ulceration and preoperatively diagnosed as 20 millimetres or less in diameter. Dig Endosc 2010;22:112-118.

14. Okada K, Fujisaki J, Yoshida T, et al. Long-term outcomes of endoscopic submucosal dissection for undifferentiated-type early gastric cancer. Endoscopy 2012;44:122-127.

15. Abe S, Oda I, Suzuki H, et al. Short- and long-term outcomes of endoscopic submucosal dissection for undifferentiated early gas- tric cancer. Endoscopy 2013;45:703-707.

16. Min BH, Kang KJ, Lee JH, et al. Endoscopic resection for undifferentiated early gastric cancer: focusing on histologic discrepancies between forceps biopsy-based and endoscopic resection specimenbased diagnosis. Dig Dis Sci 2014;59:2536-2543.

17. Japanese Gastric Cancer Association. Japanese classification of gastric carcinoma: 3rd English edition. Gastric Cancer 2011; 14:101-112.

18. Akahoshi K, Chijiwa Y, Hamada S, et al. Pretreatment staging of endoscopically early gastric cancer with a $15 \mathrm{MHz}$ ultrasound catheter probe. Gastrointest Endosc 1998;48:470-476.

19. Kim HM, Pak KH, Chung MJ, et al. Early gastric cancer of signet ring cell carcinoma is more amenable to endoscopic treatment than is early gastric cancer of poorly differentiated tubular adenocarcinoma in select tumor conditions. Surg Endosc 2011;25:30873093.

20. Ha TK, An JY, Youn HK, Noh JH, Sohn TS, Kim S. Indication for endoscopic mucosal resection in early signet ring cell gastric cancer. Ann Surg Oncol 2008;15:508-513.

21. Park YD, Chung YJ, Chung HY, et al. Factors related to lymph node metastasis and the feasibility of endoscopic mucosal resection for treating poorly differentiated adenocarcinoma of the stomach. Endoscopy 2008;40:7-10.

22. Yamao T, Shirao K, Ono H, et al. Risk factors for lymph node metastasis from intramucosal gastric carcinoma. Cancer 1996;77:602606.

23. Ye BD, Kim SG, Lee JY, et al. Predictive factors for lymph node metastasis and endoscopic treatment strategies for undifferentiated early gastric cancer. J Gastroenterol Hepatol 2008;23:46-50.

24. Oka S, Tanaka S, Higashiyama M, et al. Clinical validity of the expanded criteria for endoscopic resection of undifferentiated-type early gastric cancer based on long-term outcomes. Surg Endosc 2014;28:639-647.

25. Kim JH, Kim YH, Jung DH, et al. Follow-up outcomes of endoscopic resection for early gastric cancer with undifferentiated histology. Surg Endosc 2014;28:2627-2633.

26. Choi J, Kim SG, Im JP, Kim JS, Jung HC, Song IS. Comparison of endoscopic ultrasonography and conventional endoscopy for prediction of depth of tumor invasion in early gastric cancer. Endoscopy 2010;42:705-713.

27. Sawada S, Fujisaki J, Yamamoto N, et al. Expansion of indications for endoscopic treatment of undifferentiated mucosal gastric cancer: analysis of intramucosal spread in resected specimens. Dig Dis Sci 2010;55:1376-1380.

28. Nagahama T, Yao K, Maki S, et al. Usefulness of magnifying endoscopy with narrow-band imaging for determining the horizontal extent of early gastric cancer when there is an unclear margin by chromoendoscopy (with video). Gastrointest Endosc 2011;74:1259-1267.

29. Ren G, Cai R, Zhang WJ, Ou JM, Jin YN, Li WH. Prediction of risk factors for lymph node metastasis in early gastric cancer. World $\mathrm{J}$ Gastroenterol 2013;19:3096-3107. 
30. Boku T, Nakane Y, Okusa T, et al. Strategy for lymphadenectomy of gastric cancer. Surgery 1989;105:585-592.

31. Seto Y, Shimoyama S, Kitayama J, et al. Lymph node metastasis and preoperative diagnosis of depth of invasion in early gastric cancer. Gastric Cancer 2001;4:34-38.

32. Lee E, Chae Y, Kim I, Choi J, Yeom B, Leong AS. Prognostic relevance of immunohistochemically detected lymph node micrometastasis in patients with gastric carcinoma. Cancer 2002;94:28672873.

33. Choi HJ, Kim YK, Kim YH, Kim SS, Hong SH. Occurrence and prognostic implications of micrometastases in lymph nodes from patients with submucosal gastric carcinoma. Ann Surg Oncol 2002;9:13-19.

34. Hyung WJ, Cheong JH, Kim J, Chen J, Choi SH, Noh SH. Application of minimally invasive treatment for early gastric cancer. $\mathrm{J}$ Surg Oncol 2004;85:181-185.

35. Abe N, Watanabe T, Sugiyama M, et al. Endoscopic treatment or surgery for undifferentiated early gastric cancer? Am J Surg 2004;188:181-184.

36. Pyo JH, Shin CM, Lee H, et al. A risk-prediction model based on lymph-node metastasis for incorporation into a treatment algorithm for signet ring cell-type intramucosal gastric cancer. Ann Surg 2016;264:1038-1043.

37. Pyo JH, Lee H, Min BH, et al. A risk prediction model based on lymph-node metastasis in poorly differentiated-type intramucosal gastric cancer. PLoS One 2016;11:e0156207.

38. Hirasawa T, Fujisaki J, Fukunaga T, et al. Lymph node metastasis from undifferentiated-type mucosal gastric cancer satisfying the expanded criteria for endoscopic resection based on routine histological examination. Gastric Cancer 2010;13:267-270.

39. Nasu J, Hori S, Asagi A, et al. A case of small undifferentiated intramucosal gastric cancer with lymph node metastasis. Gastric Cancer 2010;13:264-266. 